TRANSACTIONS OF THE

AMERICAN MATHEMATICAL SOCIETY

Volume 362, Number 11, November 2010, Pages 5845-5859

S 0002-9947(2010)05078-0

Article electronically published on June 10, 2010

\title{
POINCARÉ RECURRENCE FOR OBSERVATIONS
}

\author{
JÉRÔME ROUSSEAU AND BENOÎT SAUSSOL
}

\begin{abstract}
A high dimensional dynamical system is often studied by experimentalists through the measurement of a relatively low number of different quantities, called an observation. Following this idea and in the continuity of Boshernitzan's work, for a measure preserving system we study Poincaré recurrence for the observation. The link between the return time for the observation and the Hausdorff dimension of the image of the invariant measure is considered. We prove that when the decay of correlations is super polynomial, the recurrence rates for the observations and the pointwise dimensions relative to the push-forward are equal.
\end{abstract}

\section{INTRODUCTION}

The well-known Zermelo paradox reveals that the classical Poincaré recurrence theorem has implications in contradiction with common sense. Let us indeed consider a box filled with gas and divided into two compartments; at initial state, all the particles are in one of them. When opening the separation between the compartments, the gas will obviously spread in the entire box. The Poincaré recurrence theorem states that the gas will come back arbitrarily close to its initial state, i.e. in the original compartment. But noboody will ever see this event happening (since the time needed to come back is greater than the lifetime of the galaxy). Nevertheless, if we focus on a fewer number of these particles, this event will appear after a reasonable time. In the same way, when we study a high dimensional dynamical system we might not know all the aspects of the evolution, but instead only a part or certain quantities of the system. This might be due to the difficulty of studying a high dimensional system, but it also might be the lack of interest of an over-detailed description.

Recently, Ott and York tried to elaborate some Platonic formalism of dynamical systems [11. The reality, the dynamical system $(X, T, \mu)$, is only known through a measurement or observation, that is, a function defined on $X$ taking values in (typically) a lower dimensional space. The following result by Boshernitzan 4] about Poincaré recurrence falls into this frame. If we have a measure preserving dynamical system $(X, T, \mu)$ and an observable $f$ from $X$ to a metric space $(Y, d)$, then whenever the $\alpha$-dimensional Hausdorff measure is $\sigma$-finite on $Y$ we have

$$
\liminf _{n \rightarrow \infty} n^{1 / \alpha} d\left(f(x), f\left(T^{n} x\right)\right)<\infty \quad \text { for } \mu \text {-almost every } x .
$$

Received by the editors July 7, 2008.

2010 Mathematics Subject Classification. Primary 37C45, 37B20; Secondary 37A25, 37DXX, $37 \mathrm{M} 25$.

Key words and phrases. Poincaré recurrence, dimension theory, decay of correlations.

(C)2010 American Mathematical Society Reverts to public domain 28 years from publication 
The main aim of this paper is to prove a refinement of (1D) and a generalization of [2, 12] for recurrence rates for observations.

In Section 2 we give the precise definition of the recurrence rates for the observations and state an upper bound in terms of dimension (Theorem 2 which is proved in Section 3), then under an additional assumption we state our main result (Theorem 5 which is proved in Section 4), and finally, we analyze in the case of the Lebesgue measure the existence of the pointwise dimension for its smooth image (Theorem 9 which is proved in Section 5).

\section{Statement of the Results}

2.1. Definitions and general inequality. Let $(X, \mathcal{A}, \mu, T)$ be a measure preserving system (m.p.s.), i.e. $\mathcal{A}$ is a $\sigma$-algebra, $\mu$ is a measure on $(X, \mathcal{A})$ with $\mu(X)=1$ and $\mu$ is invariant by $T$ (i.e. $\mu\left(T^{-1} A\right)=\mu(A)$ for all $A \in \mathcal{A}$ ), where $T: X \rightarrow X$.

Let $f: X \rightarrow Y$ be a function called observable (we will specify the space $X$ and $Y$ later). We introduce the return time for the observation and its associated recurrence rates.

Definition 1. Let $f: X \rightarrow Y$ be a measurable function. We define for $x \in X$ the return time for the observation

$$
\tau_{r}^{f}(x):=\inf \left\{k \in \mathbb{N}^{*}: f\left(T^{k} x\right) \in B(f(x), r)\right\},
$$

where $B(x, r)$ is the ball centered in $x$ with radius $r$. We then define the lower and upper recurrence rate for the observation

$$
\underline{R}_{i}^{f}(x):=\liminf _{r \rightarrow 0} \frac{\log \tau_{r}^{f}(x)}{-\log r}, \quad \bar{R}_{i}^{f}(x):=\limsup _{r \rightarrow 0} \frac{\log \tau_{r}^{f}(x)}{-\log r} .
$$

We also define for $p \in \mathbb{N}$ the $p$-non-instantaneous return time for the observation

$$
\tau_{r, p}^{f}(x):=\inf \left\{k>p: f\left(T^{k} x\right) \in B(f(x), r)\right\} .
$$

Then we define the non-instantaneous lower and upper recurrence rates for the observation

$$
\underline{R}^{f}(x):=\lim _{p \rightarrow \infty} \liminf _{r \rightarrow 0} \frac{\log \tau_{r, p}^{f}(x)}{-\log r}, \quad \bar{R}^{f}(x):=\lim _{p \rightarrow \infty} \limsup _{r \rightarrow 0} \frac{\log \tau_{r, p}^{f}(x)}{-\log r} .
$$

Whenever $\underline{R}^{f}(x)=\bar{R}^{f}(x)$ we denote by $R^{f}(x)$ the value of the limit.

The lower and upper pointwise or local dimension of a Borel probability measure $\nu$ on $Y$ at a point $y \in Y$ are defined by

$$
\underline{d}_{\nu}(y)=\varliminf_{r \rightarrow 0} \frac{\log \nu(B(y, r))}{\log r} \quad \text { and } \quad \bar{d}_{\nu}(y)=\varlimsup_{r \rightarrow 0} \frac{\log \nu(B(y, r))}{\log r} .
$$

The push-forward measure $f_{*} \mu():.=\mu\left(f^{-1}().\right)$ is a probability measure on $Y$, and we define the lower and upper pointwise dimension for the observations with respect to $\mu$ at a point $x \in X$ by

$$
\underline{d}_{\mu}^{f}(x)=\underline{d}_{f_{*} \mu}(f(x)) \quad \text { and } \quad \bar{d}_{\mu}^{f}(x)=\bar{d}_{f_{*} \mu}(f(x)) .
$$

If they are equal, we denote by $d_{\mu}^{f}(x)$ the common value. 
Theorem 2. Let $(X, \mathcal{A}, \mu, T)$ be an m.p.s. Consider a measurable observable $f$ : $X \rightarrow Y=\mathbb{R}^{N}$. Then

$$
\underline{R}^{f}(x) \leq \underline{d}_{\mu}^{f}(x) \quad \text { and } \quad \bar{R}^{f}(x) \leq \bar{d}_{\mu}^{f}(x)
$$

for $\mu$-almost every $x \in X$.

This result is satisfactory in the sense that it holds for any dynamical system and observation. Moreover, under natural assumptions we will show that the equality is true. Still, these inequalities may be strict; the caricatural example occurs when $T$ is the identity map.

Example 3. Let $(\Omega, \mathcal{F}, \mathbb{P})$ be a probability space together with a $\mathbb{P}$-preserving map $\theta$ and let $Y \subset \mathbb{R}^{N}$ be a Borel set. The family $\left(F_{\omega}\right)_{\omega \in \Omega}$ is called a random transformation, where for each $\omega, F_{\omega}$ is a map from $Y$ to $Y$ such that the map $(w, y) \rightarrow F_{\omega}(y)$ is $\mathcal{F} \times \mathcal{B}\left(\mathbb{R}^{N}\right)$-measurable. The map $T: X=\Omega \times Y \rightarrow X$ defined by $T(\omega, y)=\left(\theta \omega, F_{\omega}(y)\right)$ is called a skew product transformation. Let $\mathcal{M}_{\mathbb{P}}(X, T)$ be the set of $T$-invariant probability measure having the marginal $\mathbb{P}$ on $\Omega$. For any $\mu \in \mathcal{M}_{\mathbb{P}}(X, T)$, Theorem 2 applies with $f$ the projection on $Y$, and it gives an upper bound for the time needed by a typical random orbit $F_{\theta^{k} \omega} \circ \ldots \circ F_{\theta \omega} \circ F_{\omega}(y)$ to come back close to its starting point $y$.

2.2. Poincaré recurrence for observations. From now on let us assume that $X$ is a metric space and $\mathcal{A}$ is its Borel $\sigma$-algebra. We then can introduce the decay of correlations:

Definition 4. $(X, T, \mu)$ has a super-polynomial decay of correlations if, for all $\phi$, $\psi$ Lipschitz functions from $X$ to $\mathbb{R}$ and for all $n \in \mathbb{N}^{*}$, we have

$$
\left|\int_{X} \phi \circ T^{n} \psi d \mu-\int_{X} \phi d \mu \int_{X} \psi d \mu\right| \leq\|\phi\|\|\psi \psi\| \theta_{n}
$$

with $\lim _{n \rightarrow \infty} \theta_{n} n^{p}=0$ for all $p>0$ and where $\|$.$\| is the Lipschitz norm.$

The main result of our paper is:

Theorem 5. Let $(X, \mathcal{A}, \mu, T)$ be an m.p.s. with a super-polynomial decay of correlations. Consider a Lipschitz observable $f: X \rightarrow Y=\mathbb{R}^{N}$. Then, we have

$$
\underline{R}^{f}(x)=\underline{d}_{\mu}^{f}(x) \quad \text { and } \quad \bar{R}^{f}(x)=\bar{d}_{\mu}^{f}(x)
$$

for $\mu$-almost every $x$ such that $\underline{d}_{\mu}^{f}(x)>0$.

Taking the identity function for $f$, we recover the result of [2] and [12] under weaker assumptions. The main assumption of the theorem about decay of correlations is satisfied in a variety of systems with some hyperbolic behavior and studied in abundant literature (e.g. [13, 5, 1,).

Definition 6. We say that a probability measure $\nu$ is exact dimensional if there exists a constant $d_{\nu} \in \mathbb{R}$ such that

$$
\underline{d}_{\nu}(\cdot)=\bar{d}_{\nu}(\cdot)=d_{\nu} \quad \text { almost everywhere. }
$$

It is well known that in this case many notions of dimension coincide (see Section 5.1 for details). In particular the Hausdorff dimension $\operatorname{dim}_{H} \nu$ satisfies

Proposition 7. If $\nu$ is exact dimensional, then

$$
d_{\nu}(\cdot)=\operatorname{dim}_{H} \nu \text { almost everywhere. }
$$


Corollary 8. Let $(X, \mathcal{A}, \mu, T)$ be an m.p.s. with a super-polynomial decay of correlations. Consider a Lipschitz observable $f: X \rightarrow Y=\mathbb{R}^{N}$. Then, if $f_{*} \mu$ is exact dimensional, we have

$$
\underline{R}^{f}(x)=\bar{R}^{f}(x)=\operatorname{dim}_{H} f_{*} \mu \quad \text { for } \mu \text {-almost every } x \in X .
$$

Remark. We have the equivalence

$f_{*} \mu$ is exact dimensional $\Longleftrightarrow \exists d, \underline{d}_{\mu}^{f}(x)=\bar{d}_{\mu}^{f}(x)=d \quad$ for $\mu$-almost every $x \in X$.

Proof of Corollary 8, If $\operatorname{dim}_{H} f_{*} \mu=0$, then the conclusion follows from Theorem 2 and Proposition 7 . In the general case, it is just a combination of Theorem 5 and Proposition 7

Theorem 5 does not apply to those points where $\underline{d}_{\mu}^{f}(x)=0$. When $\bar{d}_{\mu}^{f}(x)=0$ also, this is not a restriction because Theorem 2 applies and gives $\bar{R}^{f}(x)=\underline{R}^{f}(x)=0$. However, the question remains when $\bar{d}_{\mu}^{f}(x) \neq \underline{d}_{\mu}^{f}(x)=0$ on a positive measure set. Indeed, the assumptions of Theorem 5 are not strong enough to ensure the almost everywhere existence of the pointwise dimension for the observations. The following result guarantees the existence for a large class of systems.

Theorem 9. Let $f: \mathbb{R}^{M} \rightarrow \mathbb{R}^{N}$ be a $C^{\infty}$ function, and let $\mu$ be any absolutely continuous measure on $\mathbb{R}^{M}$. Then, $d_{\mu}^{f}$ exists and belongs to $\{0,1, \ldots, \min \{M, N\}\}$ $\mu$-almost everywhere. More precisely, $d_{\mu}^{f}(x)=\operatorname{rank} d_{x} f$ for $\mu$-almost every $x \in \mathbb{R}^{M}$.

This is a non-trivial result because the image measure $f_{*} \mu$ may be quite complicated and rather counterintuitive. Already in the one dimensional case there exists $f \in C^{\infty}(\mathbb{R}, \mathbb{R})$ such that $f\left(\left\{f^{\prime}=0\right\}\right)$ is an uncountable set of dimension 0 and $f_{*}\left(\left.L e b\right|_{\left\{f^{\prime}=0\right\}}\right)$ is a non-null and non-atomic measure. We emphasize that Theorem 9 applies to any $C^{\infty}$ function, and not only for generic functions. This is essential in applications where we are most interested in particular observables.

Corollary 10. Let $T: X \subset \mathbb{R}^{M} \rightarrow X$ preserve an absolutely continuous invariant probability measure with super-polynomial decay of correlations. Let $f: \mathbb{R}^{M} \rightarrow \mathbb{R}^{N}$ be a Lipschitz $C^{\infty}$ observable. Then $R^{f}$ exists and belongs to $\{0,1, \ldots, \min \{M, N\}\}$ almost everywhere.

Proof of Corollary 10. We apply Theorem 5 and Theorem 9 when $d_{\mu}^{f}>0$. When $d_{\mu}^{f}=0$, we use Theorem 2,

2.3. On the necessity of the non-instantaneous recurrence rate. In this part, we give a simple example which illustrates the utility of non-instantaneous return times.

Let $\Omega:=\{0,1\}^{\mathbb{N}}$ and $\sigma$ be the shift on $\Omega$. Fix some 1-approximable $\alpha \in \mathbb{R}$ (e.g. [6] for a nice perspective), i.e. $\delta(\alpha)=1$, where

$$
\delta(\alpha)=\sup \left\{\delta \geq 1:\left|\alpha-\frac{p}{q}\right|<\frac{1}{q^{1+\delta}} \text { for infinitely many } \frac{p}{q} \in \mathbb{Q}\right\} .
$$

Let $\nu$ be an invariant ergodic probability measure on $\Omega$. Fix some measurable $A \subset \Omega$ such that $1>\nu(A)>0$ and set $\varphi$ :

$$
\varphi(\omega)= \begin{cases}0 & \text { if } \omega \notin A \\ \alpha & \text { if } \omega \in A\end{cases}
$$


Let $\mathbb{T}^{1}$ denote the one-dimensional torus and define on $X:=\Omega \times \mathbb{T}^{1}$ the map

$$
\begin{aligned}
T: X & \longrightarrow X, \\
(\omega, y) & \longrightarrow(\sigma \omega, y+\varphi(\omega)) .
\end{aligned}
$$

Let $L e b$ be the Lebesgue measure on $\mathbb{T}^{1}$. The probability measure $\mu:=\nu \otimes L e b$ is $T$-invariant. We examine below the recurrence rate of the system $(X, T, \mu)$ for the observable $f$ given by the projection on the second variable, i.e.

$$
\begin{aligned}
& f: \quad X \rightarrow \mathbb{T}^{1}, \\
& (\omega, y) \rightarrow y \text {. }
\end{aligned}
$$

First, we need the following obvious result on the push-forward measure: since $f_{*} \mu=L e b$ and the local dimension of the Lebesgue measure is one, the measure $f_{*} \mu$ is exact dimensional and satisfies

$$
\forall x \in X, d_{\mu}^{f}(x)=1 .
$$

Proposition 11. We have $R_{i}^{f} \neq d_{\mu}^{f}$ on a set of positive measure. More precisely,

$$
\forall x=(\omega, y) \in \Omega \backslash A \times \mathbb{T}^{1}, R_{i}^{f}(x)=0 .
$$

Proof. Let $\omega \in \Omega \backslash A$ and $y \in \mathbb{T}^{1}$; we have

$$
\begin{aligned}
f(T(\omega, y)) & =f(\sigma \omega, y) \quad \text { because } \omega \notin A \\
& =y \\
& =f(\omega, y) .
\end{aligned}
$$

So, for all $r>0, \tau_{r}^{f}(x)=1$, and then $R_{i}^{f}(x)=0$.

We therefore need to introduce the non-instantaneous return time to avoid this kind of problem.

Proposition 12. We have $R^{f}=d_{\mu}^{f}$ on a set of full measure.

Proof. For $k \in \mathbb{N}$ and $\omega \in \Omega$, let $q_{k}(\omega):=\sum_{i=0}^{k-1} \mathbf{1}_{A}\left(\sigma^{i} \omega\right)$. Let $\varepsilon>0$ fixed. For $x=(\omega, y)$ and $n \in \mathbb{N}$, we have

$$
\begin{aligned}
\tau_{\frac{1}{n^{1+\varepsilon}}, p}^{f}(x) & =\inf \left\{k>p: f\left(T^{k} x\right) \in B\left(f(x), \frac{1}{n^{1+\varepsilon}}\right)\right\} \\
& =\inf \left\{k>p: y+\alpha q_{k}(\omega) \in B\left(y, \frac{1}{n^{1+\varepsilon}}\right)\right\} \\
& =\inf \left\{k>p:\left\|\alpha q_{k}(\omega)\right\| \leq \frac{1}{n^{1+\varepsilon}}\right\},
\end{aligned}
$$

where for $q \in \mathbb{Z}$

$$
\|q \alpha\|:=\min \{|q \alpha-p|: p \in \mathbb{Z}\} .
$$

Thanks to the choice of $\alpha$, there exists $k_{0} \in \mathbb{N}$ such that for all $k \geq k_{0}$, we get $\|k \alpha\| \geq \frac{1}{k^{1+\varepsilon}}$. Taking $n \geq k_{0}$ we have

$$
m_{n, \varepsilon}:=\inf \left\{q>k_{0}:\|q \alpha\| \leq \frac{1}{n^{1+\varepsilon}}\right\} \geq n .
$$

Since $\nu$ is ergodic, the Poincaré Recurrence Theorem gives, for $\nu$-almost every $\omega \in \Omega$,

$$
q_{k}(\omega) \underset{k \rightarrow \infty}{\longrightarrow}+\infty
$$


So, for $\nu$-almost every $\omega \in \Omega$, we can choose $p$ sufficiently large such that $p \geq k_{0}$ and $q_{p}(\omega) \geq k_{0}$. If $k \in \mathbb{N}$ satisfies $p \leq k<m_{n, \varepsilon}$, then $k_{0} \leq q_{p}(\omega) \leq q_{k}(\omega) \leq$ $k<m_{n, \varepsilon}$, and so $\left\|\alpha q_{k}(\omega)\right\|>\frac{1}{n^{1+\varepsilon}}$. Since this is true for every $k \in\left[p, \ldots, m_{n, \varepsilon}\right]$ we end up with $\tau_{\frac{1}{n^{1+\varepsilon}}, p}^{f}(x) \geq m_{n, \varepsilon} \geq n$. Finally, if $p \geq m_{n, \varepsilon}$, we obviously have $\tau_{\frac{1}{n^{1+\varepsilon}}, p}^{f}(x) \geq m_{n, \varepsilon} \geq n$. Thus for $\mu$-almost every $x=(\omega, y) \in X$, we have

$$
\begin{aligned}
\underline{R}^{f}(x) & =\lim _{p \rightarrow \infty} \liminf _{n \rightarrow+\infty} \frac{\log \tau_{\frac{1}{n^{1+\varepsilon}}, p}^{f}(x)}{-\log \frac{1}{n^{1+\varepsilon}}} \\
& \geq \liminf _{n \rightarrow+\infty} \frac{\log m_{n, \varepsilon}}{-\log \frac{1}{n^{1+\varepsilon}}} \\
& \geq \lim _{n \rightarrow \infty} \frac{\log n}{\log n^{1+\varepsilon}}=\frac{1}{1+\varepsilon} .
\end{aligned}
$$

This is true for all $\varepsilon>0$; thus

$$
\underline{R}^{f}(x) \geq 1 .
$$

The conclusion follows from Theorem 2 and equation (3).

Remark. We point out that indeed our example fulfills the conditions of Corollary 8 when, for example, $\nu$ is a Gibbs measure [7].

\section{UPPER BOUND FOR THE RECURRENCE RATE IN MEASURE PRESERVING SYSTEMS}

The basic strategy of the proof of Theorem 2 follows 2 . We recall the definition of a weakly diametrically regular measure.

Definition 13. A measure $\mu$ is weakly diametrically regular (wdr) on the set $Z \subset X$ if for any $\eta>1$, for $\mu$-almost every $x \in Z$ and every $\varepsilon>0$, there exists $\delta>0$ such that if $r<\delta$, then $\mu(B(x, \eta r)) \leq \mu(B(x, r)) r^{-\varepsilon}$.

Proof of Theorem 2. Since any probability measure is weakly diametrically regular on $\mathbb{R}^{N}$ [2] the measure $f_{*} \mu$ is weakly diametrically regular. We can remark that the function $\delta(f(\cdot), \varepsilon, \eta)$ in the previous definition can be made measurable for every fixed $\varepsilon$ and $\eta$. Let us fix $\varepsilon>0$ and $\eta=4$. We choose $\delta>0$ sufficiently small to have $\mu(G)>\mu(X)-\varepsilon=1-\varepsilon$, where

$$
G:=\{x \in X: \delta(f(x), \varepsilon, \eta)>\delta\} .
$$

For all $r>0, \lambda>0, p \in \mathbb{N}$ and $x \in X$ we define the set

$$
A_{r, x}:=\left\{y \in X: f(y) \in B(f(x), 4 r), \tau_{4 r, p}^{f}(y, x) \geq \lambda^{-1} f_{*} \mu(B(f(x), 4 r))^{-1}\right\},
$$

where $\tau_{4 r, p}^{f}(y, x):=\inf \left\{k>p: d\left(f\left(T^{k} y\right), f(x)\right)<4 r\right\}$ for $y \in f^{-1} B(f(x), 4 r)$. Markov's inequality gives

$$
\mu\left(A_{r, x}\right) \leq \lambda f_{*} \mu(B(f(x), 4 r)) \int_{f^{-1} B(f(x), 4 r)} \tau_{4 r, p}^{f}(y, x) d \mu(y) .
$$

Since $\tau_{4 r, p}^{f}(y, x)$ is bounded by the $p^{t h}$ return time of $y$ in the set $f^{-1} B(f(x), 4 r)$, by Kac's lemma we have

$$
\int_{f^{-1} B(f(x), 4 r)} \tau_{4 r, p}^{f}(y, x) d \mu(y) \leq p
$$


Using (7) and (8), we have

$$
\mu\left(A_{r, x}\right) \leq p \lambda f_{*} \mu(B(f(x), 4 r)) .
$$

If $d(f(x), f(y))<2 r$, then

$$
\tau_{4 r, p}^{f}(y, x) f_{*} \mu(B(f(x), 4 r)) \geq \tau_{6 r, p}^{f}(y) f_{*} \mu(B(f(y), 2 r)) .
$$

Definition 14. Given $r>0$, a countable set $E \subset F$ is a maximal $r$-separated set for $F$ if

(1) $B\left(x, \frac{r}{2}\right) \cap B\left(y, \frac{r}{2}\right)=\emptyset$ for any two distinct $x, y \in E$.

(2) $\mu\left(F \backslash \bigcup_{x \in E} B(x, r)\right)=0$.

Let $C \subset G$ such that $(f(x))_{x \in C}$ is a maximal $2 r$-separated set for $f(G)$ :

$$
\begin{aligned}
D_{\varepsilon}(r) & :=\mu\left(\left\{y \in G: \tau_{6 r, p}^{f}(y) f_{*} \mu(B(f(y), 2 r)) \geq r^{-2 \varepsilon}\right\}\right) \\
& \leq \sum_{x \in C} \mu\left(\left\{y \in f^{-1} B(f(x), 2 r): \tau_{6 r, p}^{f}(y) f_{*} \mu(B(f(y), 2 r)) \geq r^{-2 \varepsilon}\right\}\right) \\
& \leq \sum_{x \in C} \mu\left(A_{r, x}\right) \quad \text { by (10) } \\
& \leq p r^{2 \varepsilon} \sum_{x \in C} f_{*} \mu(B(f(x), 4 r)) \quad \text { with } \lambda=r^{2 \varepsilon} \text { in (9) } \\
& \leq p r^{\varepsilon} \sum_{x \in C} f_{*} \mu(B(f(x), r)) \quad \text { since } f_{*} \mu \text { is wdr and with } \eta=4 \\
& \leq p r^{\varepsilon} \quad \text { according to the definition of } C .
\end{aligned}
$$

Finally,

$$
\sum_{n, e^{-n}<\delta} D_{\varepsilon}\left(e^{-n}\right)=\sum_{n>-\log \delta} D_{\varepsilon}\left(e^{-n}\right) \leq p \sum_{n} e^{-\varepsilon n}<\infty .
$$

Then, thanks to the Borel-Cantelli lemma, for $\mu$-almost every $x \in G$

$$
\tau_{6 e^{-n}, p}^{f}(x) f_{*} \mu\left(B\left(f(x), 2 e^{-n}\right)\right) \leq e^{2 \varepsilon n},
$$

for any $n$ sufficiently large. Then

$$
\frac{\log \tau_{6 e^{-n}, p}^{f}(x)}{n} \leq 2 \varepsilon+\frac{\log f_{*} \mu\left(B\left(f(x), 2 e^{-n}\right)\right)}{-n} .
$$

Observing that for all $a>0$ we have

$$
\begin{gathered}
\underline{d}_{\mu}^{f}(x)=\varliminf_{n \rightarrow \infty} \frac{\log f_{*} \mu\left(B\left(f(x), a e^{-n}\right)\right)}{-n} \text { and } \bar{d}_{\mu}^{f}(x)=\varlimsup_{n \rightarrow \infty} \frac{\log f_{*} \mu\left(B\left(f(x), a e^{-n}\right)\right)}{-n}, \\
\underline{R}^{f}(x)=\lim _{p \rightarrow \infty} \liminf _{n \rightarrow \infty} \frac{\log \tau_{a e^{-n}, p}^{f}(x)}{n} \text { and } \quad \bar{R}^{f}(x)=\lim _{p \rightarrow \infty} \limsup _{n \rightarrow \infty} \frac{\log \tau_{a e^{-n}, p}^{f}(x)}{n},
\end{gathered}
$$

and since $\varepsilon$ can be chosen arbitrarily small, we have the result if we take the limit inferior or the limit superior and then the limit over $p$ in (11). 


\section{RECURREnCE RATE AND DIMENSION IN RAPIDLY MIXING SYSTEMS}

Despite some similarities with [12], we emphasize that the proof of Theorem 5] is relatively different. In particular, we make no assumption on the entropy of the system.

Let $a>0, b>0, c>0$ and $\rho>0$. Let $X_{a}:=\left\{x \in X, \underline{d}_{\mu}^{f}(x)>a\right\}$. Set

$$
\begin{aligned}
G_{1} & =\left\{x \in X_{a}: \forall r \leq \rho, f_{*} \mu\left(B\left(f(x), \frac{r}{2}\right)\right) \leq r^{a}\right\}, \\
G_{2} & =\left\{x \in X_{a}: \forall r \leq \rho, f_{*} \mu\left(B\left(f(x), \frac{r}{2}\right)\right) \geq r^{N+b}\right\}, \\
G_{3} & =\left\{x \in X_{a}: \forall r \leq \rho, f_{*} \mu\left(B\left(f(x), \frac{r}{2}\right)\right) \geq f_{*} \mu(B(f(x), 4 r)) r^{c}\right\} .
\end{aligned}
$$

We observe that $G(a, b, c, \rho):=G_{1} \cap G_{2} \cap G_{3}$ satisfies

$$
\mu(G(a, b, c, \rho)) \underset{\rho \rightarrow 0}{\longrightarrow} \mu\left(X_{a}\right) .
$$

Indeed, by definition of $\underline{d}_{\mu}^{f}$, we have $\mu\left(G_{1}\right) \rightarrow \mu\left(X_{a}\right)$. Moreover, since $\bar{d}_{\mu}^{f} \leq N$ $\mu$-almost everywhere, $\mu\left(G_{2}\right) \rightarrow \mu\left(X_{a}\right)$, and since the measure $f_{*} \mu$ is weakly diametrically regular, $\mu\left(G_{3}\right) \rightarrow \mu\left(X_{a}\right)$.

Lemma 15. Under the assumptions of Theorem [5, for any $x \in G(a, b, c, \rho)$, for any $n \in \mathbb{N}^{*}$ and for any $r \leq \rho$ we have

$$
\mu\left(f^{-1} B(f(x), r) \cap T^{-n} f^{-1} B(f(x), 2 r)\right) \leq \frac{L^{2}}{r^{2}} \theta_{n}+r^{-2 c} f_{*} \mu\left(B\left(f(x), \frac{r}{2}\right)\right)^{2} .
$$

Proof. Let $\eta_{r}:[0,+\infty) \rightarrow \mathbb{R}$ be the $\frac{1}{r}$-Lipschitz function such that $1_{[0, r]} \leq \eta_{r} \leq$ $1_{[0,2 r]}$. Set $\psi_{x, r}(y)=\eta_{r}(d(f(x), f(y)))$. Since $f$ is $L$-Lipschitz, $\psi_{x, r}$ is clearly $\frac{L}{r}$-Lipschitz. Using the assumption on the decay of correlations of $(X, T, \mu)$, we obtain

$$
\begin{aligned}
& \mu\left(f^{-1} B(f(x), r) \cap T^{-n} f^{-1} B(f(x), 2 r)\right) \leq \int_{X} \psi_{x, r}(y) \psi_{x, 2 r}\left(T^{n} y\right) d \mu(y) \\
& \leq\left\|\psi_{x, r}\right\|\left\|\psi_{x, 2 r}\right\| \theta_{n}+\int_{X} \psi_{x, r}(y) d \mu(y) \int_{X} \psi_{x, 2 r}(y) d \mu(y) \\
& \leq \frac{L^{2}}{r^{2}} \theta_{n}+f_{*} \mu(B(f(x), 2 r)) f_{*} \mu(B(f(x), 4 r)) \\
& \leq \frac{L^{2}}{r^{2}} \theta_{n}+r^{-2 c} f_{*} \mu\left(B\left(f(x), \frac{r}{2}\right)\right)^{2} .
\end{aligned}
$$

Lemma 16. Under the assumptions of Theorem 5 ,

$$
\underline{R}^{f}(x)>0 \text { for } \mu \text {-almost every } x \text { such that } \underline{d}_{\mu}^{f}(x)>0 \text {. }
$$

Proof. Let $X_{+}:=\left\{\underline{d}_{\mu}^{f}>0\right\}$. Let $1>\varepsilon>0$ and let $a>0$ such that $\mu\left(X_{+}\right) \geq$ $\mu\left(X_{a}\right)>\mu\left(X_{+}\right)-\varepsilon$.

We fix $b>0, c=\frac{a}{4}$, and for $\rho>0$ we consider the set $G=G(a, b, c, \rho)$ defined above.

Let $n_{0} \in \mathbb{N}$ such that $\forall n \geq n_{0}$, we have $\varepsilon_{n}=\frac{1}{n^{4 / a}}<\rho$. For $n \geq n_{0}$ we define

$$
A_{n}:=\left\{x \in X: f\left(T^{n} x\right) \in B\left(f(x), \varepsilon_{n}\right)\right\} .
$$


For any $x \in G$ we have

$$
f^{-1} B\left(f(x), \varepsilon_{n}\right) \cap A_{n} \subset f^{-1} B\left(f(x), \varepsilon_{n}\right) \cap T^{-n} f^{-1} B\left(f(x), 2 \varepsilon_{n}\right) .
$$

By Lemma 15] and the definition of $G$ we have

$$
\begin{aligned}
\mu\left(f^{-1} B\left(f(x), \varepsilon_{n}\right) \cap A_{n}\right) & \leq \frac{L^{2}}{\varepsilon_{n}^{2}} \theta_{n}+\varepsilon_{n}^{-2 c} f_{*} \mu\left(B\left(f(x), \frac{\varepsilon_{n}}{2}\right)\right)^{2} \\
& \leq f_{*} \mu\left(B\left(f(x), \frac{\varepsilon_{n}}{2}\right)\right)\left[L^{2}\left(\varepsilon_{n}\right)^{-2-N-b} \theta_{n}+\varepsilon_{n}^{a / 2}\right] .
\end{aligned}
$$

Let $B \subset G$ such that $(f(x))_{x \in B}$ is a maximal $\varepsilon_{n}$-separated set for $f(G)$. Since the collection $\left(f^{-1} B\left(f(x), \varepsilon_{n}\right)\right)_{x \in B}$ covers $G$, we have

$$
\begin{aligned}
\mu\left(G \cap A_{n}\right) & \leq \sum_{x \in B} \mu\left(f^{-1} B\left(f(x), \varepsilon_{n}\right) \cap A_{n}\right) \\
& \leq \sum_{x \in B} f_{*} \mu\left(B\left(f(x), \frac{\varepsilon_{n}}{2}\right)\right)\left[L^{2}\left(\varepsilon_{n}\right)^{-2-N-b} \theta_{n}+\varepsilon_{n}^{a / 2}\right] \\
& \leq L^{2}\left(\varepsilon_{n}\right)^{-2-N-b} \theta_{n}+\varepsilon_{n}^{a / 2} .
\end{aligned}
$$

Since $\sum_{n \in \mathbb{N}^{*}} \varepsilon_{n}^{a / 2}=\sum_{n \in \mathbb{N}^{*}} \frac{1}{n^{2}}<\infty$ and since the decay of correlations is superpolynomial, we obtain

$$
\sum_{n \in \mathbb{N}^{*}} \mu\left(G \cap A_{n}\right)<+\infty .
$$

By the Borel-Cantelli lemma and using (12), we have that for $\mu$-almost every $x \in$ $X_{a}$, there exists $n_{1}(x)$ such that for every $n \geq n_{1}(x), f\left(T^{n} x\right) \notin B\left(f(x), \frac{1}{n^{4 / a}}\right)$. So, for $\mu$-almost every $x \in X_{a}$, for $p \geq n_{1}(x)$ and $n \geq n_{1}(x)$,

$$
\tau_{\frac{1}{n^{4 / a}, p}}^{f}(x)>n,
$$

which gives us

$$
\begin{aligned}
\underline{R}^{f}(x) & =\lim _{p \rightarrow \infty} \liminf _{r \rightarrow 0} \frac{\log \tau_{r, p}^{f}(x)}{-\log r} \\
& =\lim _{p \rightarrow \infty} \liminf _{n \rightarrow+\infty} \frac{\log \tau_{\frac{1}{n^{4 / a}}, p}^{f}(x)}{-\log \frac{1}{n^{4 / a}}} \\
& \geq \lim _{n \rightarrow+\infty} \frac{\log n}{\log n^{4 / a}}=\frac{a}{4}>0 .
\end{aligned}
$$

Since we can choose $\varepsilon$ arbitrarily small, the lemma is proved.

Lemma 17. Let $a>0, \delta>0$ and $1>\varepsilon>0$. For $\mu$-almost every $x \in X_{a}$, there exists $r(x)>0$ such that for every $r \in] 0, r(x)$ [ and for every integer $n \in$ $\left[r^{-\delta}, f_{*} \mu(B(f(x), r))^{-1+\varepsilon}\right]$, we have $d\left(f\left(T^{n} x\right), f(x)\right) \geq r$.

Proof. Let $1>\varepsilon>0$. We fix $b>0, c=\frac{a \varepsilon}{4}$, and for $\rho>0$ we consider the set $G=G(a, b, c, \rho)$. Let $\delta>0$ and $r \leq \rho$; we define

$A_{\varepsilon}(r):=\left\{y \in X: \exists n \in\left[r^{-\delta}, f_{*} \mu(B(f(y), 3 r))^{-1+\varepsilon}\right]\right.$ such that $\left.d\left(f\left(T^{n} y\right), f(y)\right)<r\right\}$. 
For any $x \in G$ we have

$$
\begin{aligned}
& f^{-1} B(f(x), r) \cap A_{\varepsilon}(r) \\
& =\left\{y: f(y) \in B(f(x), r), \exists n \in\left[r^{-\delta}, f_{*} \mu(B(f(y), 3 r))^{-1+\varepsilon}\right], d\left(f\left(T^{n} y\right), f(y)\right)<r\right\} \\
& \subset\left\{y: f(y) \in B(f(x), r), \exists n \in\left[r^{-\delta}, f_{*} \mu(B(f(x), 2 r))^{-1+\varepsilon}\right], d\left(f\left(T^{n} y\right), f(x)\right)<2 r\right\} \\
& =\bigcup_{r^{-\delta} \leq n \leq f_{*} \mu(B(f(x), 2 r))^{-1+\varepsilon}} f^{-1} B(f(x), r) \cap T^{-n} f^{-1} B(f(x), 2 r) .
\end{aligned}
$$

Let $k>1$ be such that $\delta(k-1)-2 \geq N+2 b$ and $\rho>0$ such that $n \geq \rho^{-\delta}$ implies $(k-1)(n+1)^{-k} \geq \theta_{n}$ (which is possible by the definition of $\theta_{n}$ ). Setting $I_{r}=\left[r^{-\delta}, f_{*} \mu(B(f(x), 2 r))^{-1+\varepsilon}\right] \cap \mathbb{N}$, we have by Lemma 15 that

$$
\begin{aligned}
\mu\left(f^{-1} B(f(x), r) \cap A_{\varepsilon}(r)\right) & \leq \sum_{n \in I_{r}}\left[\frac{L^{2}}{r^{2}} \theta_{n}+r^{-2 c} f_{*} \mu\left(B\left(f(x), \frac{r}{2}\right)\right)^{2}\right] \\
& \leq L^{2} r^{\delta(k-1)-2}+r^{-2 c} f_{*} \mu\left(B\left(f(x), \frac{r}{2}\right)\right)^{1+\varepsilon} \\
& \leq L^{2} r^{N+2 b}+r^{\frac{a \varepsilon}{2}} f_{*} \mu\left(B\left(f(x), \frac{r}{2}\right)\right) \quad \text { by definition of } G \\
& \leq f_{*} \mu\left(B\left(f(x), \frac{r}{2}\right)\right)\left(L^{2} r^{b}+r^{\frac{a \varepsilon}{2}}\right) .
\end{aligned}
$$

Let $B \subset G$ such that $(f(x))_{x \in B}$ is a maximal $r$-separated set for $f(G)$. Since the collection $\left(f^{-1} B(f(x), r)\right)_{x \in B}$ covers $G$, we have

$$
\begin{aligned}
\mu\left(G \cap A_{\varepsilon}(r)\right) & \leq \sum_{x \in B} \mu\left(f^{-1} B(f(x), r) \cap A_{\varepsilon}(r)\right) \\
& \leq \sum_{x \in B} f_{*} \mu\left(B\left(f(x), \frac{r}{2}\right)\right)\left(L^{2} r^{b}+r^{\frac{a \varepsilon}{2}}\right) \\
& \leq L^{2} r^{b}+r^{a \varepsilon / 2} .
\end{aligned}
$$

Then

$$
\sum_{k \in \mathbb{N}} \mu\left(G \cap A_{\varepsilon}\left(e^{-k}\right)\right)<+\infty .
$$

Thus, by the Borel-Cantelli lemma, we have for $\mu$-almost every $y \in G$ that there exists $n_{1}(y)$ such that for every $k \geq n_{1}(y), y \notin A_{\varepsilon}\left(e^{-k}\right)$. So, for $r$ sufficiently small there exists $k \in \mathbb{N}$ such that $e^{-k-1}<r \leq e^{-k} \leq e^{-n_{1}(y)}$. In addition, since $e^{\delta k} \leq r^{-\delta}$ and $3 e^{-k}<3 e r$, there does not exist any integer $n \in$ $\left[r^{-\delta}, f_{*} \mu(B(f(y), 3 e r))^{-1+\varepsilon}\right]$ such that $d\left(f\left(T^{n} y\right), f(y)\right)<r$. Since $f_{*} \mu$ is weakly diametrically regular, the factor $3 e$ is irrelevant and the lemma is proved.

Proof of Theorem 5. Let $\zeta>0$. Since $\underline{R}^{f}(x)>0$ for $\mu$-almost every $x \in X_{+}=$ $\left\{\underline{d}_{\mu}^{f}>0\right\}$ by Lemma 16, there exists $a>0$ such that $\mu\left(X_{+}\right) \geq \mu\left(\left\{\underline{R}^{f}>a\right\}\right)>$ $\mu\left(X_{+}\right)-\zeta$. For any $x \in\left\{\underline{R}^{f}>a\right\}$, for $p$ sufficiently large and $r$ sufficiently small, we have

$$
\tau_{r, p}^{f}(x) \geq r^{-a} .
$$

Thanks to Lemma 17 with $\delta=a$ and $\varepsilon>0$, for $\mu$-almost every $x \in\left\{\underline{R}^{f}>a\right\}$, if $r$ is sufficiently small and $p$ sufficiently large, then $\tau_{r, p}^{f}(x) \geq f_{*} \mu(B(f(x), r))^{-1+\varepsilon}$. Thus, $\underline{R}^{f} \geq(1-\varepsilon) \underline{d}_{\mu}^{f}$ and $\bar{R}^{f} \geq(1-\varepsilon) \bar{d}_{\mu}^{f} \mu$-almost everywhere on $\left\{\underline{R}^{f}>a\right\}$. 
The theorem is proved choosing $\varepsilon>0$ arbitrarily small and then $\zeta>0$ arbitrarily small.

\section{Dimensions of the smooth image of Lebesgue measure}

5.1. Hausdorff and packing dimensions. In this section we recall the notion of Hausdorff dimension, packing dimension and pointwise dimension, and the link between each other (see 9 for more details).

Let $(X, d)$ be a metric space. Let $U$ be a non-empty set; its diameter is

$$
\operatorname{diam} U:=\sup \{d(x, y): x, y \in U\} .
$$

Given $\delta>0$, a collection $\left\{U_{i}\right\}_{i \in I}$ is called a countable $\delta$-cover of a set $E$ if $I$ is countable, $E \subset \bigcup_{i \in I} U_{i}$ and for all $i \in I, 0<\operatorname{diam} U_{i} \leq \delta$.

Let $E$ be a subset of $X$ and $s \geq 0$; for $\delta>0$, we define

$$
\mathcal{H}_{\delta}^{s}(E)=\inf \left\{\sum_{i \in I}\left(\operatorname{diam} U_{i}\right)^{s}:\left\{U_{i}\right\}_{i \in I} \text { is a countable } \delta \text {-cover of } E\right\} .
$$

We then define the Hausdorff s-dimensional outer measure of $E$ as

$$
\mathcal{H}^{s}(E)=\lim _{\delta \rightarrow 0} \mathcal{H}_{\delta}^{s}(E) .
$$

There exists a unique $t$ such that $\mathcal{H}^{s}(E)=\infty$ if $s<t$ and $\mathcal{H}^{s}(E)=0$ if $s>t$, which is called the Hausdorff dimension of $E$, i.e.

$$
\operatorname{dim}_{H} E=\inf \left\{s: \mathcal{H}^{s}(E)<\infty\right\}=\sup \left\{s: \mathcal{H}^{s}(E)>0\right\} .
$$

If $\mu$ is a probability measure on $X$, we define the Hausdorff dimension of $\mu$ as

$$
\operatorname{dim}_{H} \mu=\inf \left\{\operatorname{dim}_{H} Y: \mu\left(Y^{c}\right)=0\right\} .
$$

Remark. We warn the reader that this definition of the Hausdorff dimension of a measure differs from the one given by Falconer [9], but it is the most used in ergodic theory.

Given $\varepsilon>0$, the collection $\left\{B\left(x_{i}, r_{i}\right)\right\}_{i \in I}$ is called a $\varepsilon$-packing of $E$ if $I$ is a finite or countable set, for all $i \in I$ we have $x_{i} \in E, r_{i} \leq \varepsilon$ and the balls are disjoints. For $s \geq 0$, we write

$$
\mathcal{P}_{\varepsilon}^{s}(E)=\sup \left\{\sum_{i \in I}\left(r_{i}\right)^{s}:\left\{B\left(x_{i}, r_{i}\right)\right\}_{i \in I} \text { is a } \varepsilon \text {-packing of } E\right\}
$$

and

$$
\mathcal{P}_{0}^{s}(E)=\lim _{\varepsilon \rightarrow 0} \mathcal{P}_{\varepsilon}^{s}(E) .
$$

We then introduce the s-dimensional packing outer measure,

$$
\mathcal{P}^{s}(E)=\inf \left\{\sum_{i=1}^{\infty} \mathcal{P}_{0}^{s}\left(E_{i}\right): E \subset \bigcup_{i=1}^{\infty} E_{i}\right\}
$$

and the packing dimension of $E$ is defined similar to the Hausdorff dimension,

$$
\operatorname{dim}_{P} E=\inf \left\{s: \mathcal{P}^{s}(E)<\infty\right\}=\sup \left\{s: \mathcal{P}^{s}(E)>0\right\} .
$$


For a probability measure $\mu$, we also have a packing dimension of $\mu$,

$$
\operatorname{dim}_{P} \mu=\inf \left\{\operatorname{dim}_{P} Y: \mu\left(Y^{c}\right)=0\right\} .
$$

There is a link between Hausdorff dimension, packing dimension and pointwise dimension:

Proposition 18. Assume that $X \subset \mathbb{R}^{N}$ for some $N$,

$$
\operatorname{dim}_{H} \mu=\text { ess-sup } \underline{d}_{\mu}
$$

and

$$
\operatorname{dim}_{P} \mu=\text { ess-sup } \bar{d}_{\mu}
$$

5.2. Existence of the pointwise dimension. Bates and Moreira proved a generalization of the classical Morse-Sard Theorem for Hausdorff measures. Unfortunately, in view of (22), this is not enough to get an upper bound for the upper pointwise dimension. A key ingredient of their proof is the following generalized Morse decomposition. Given a differentiable $f$ from $\mathbb{R}^{M}$ to $\mathbb{R}^{N}$, for $\kappa \in$ $\{0,1, \ldots, \min \{M, N\}\}$, we define $C_{\kappa}:=\left\{x \in \mathbb{R}^{M}: \operatorname{rank}\left(d_{x} f\right)=\kappa\right\}$.

Lemma 19 ([3]). Let $f \in C^{k}\left(\mathbb{R}^{M}, \mathbb{R}^{N}\right)$ with $k \geq 2$. Let $\kappa \leq M$ be an integer. Let $\eta>0$. There is a decomposition $\left\{A_{i}\right\}_{i \in \mathbb{N}}$ of $C_{\kappa}$ such that for each $i \in \mathbb{N}$ there exist two subspaces $E_{i}^{\prime}$ and $E_{i}^{\prime \prime}$ which satisfy $\mathbb{R}^{M}=E_{i}^{\prime} \oplus E_{i}^{\prime \prime}$, $\operatorname{dim} E_{i}^{\prime} \leq \kappa$ and if $S \subset \mathbb{R}^{M}$,

$$
\operatorname{diam}\left(f\left(S \cap A_{i}\right)\right) \leq\left(\left\|\left.f\right|_{A_{i}}\right\|_{C^{1}}+\eta\right) \operatorname{diam}\left(\pi_{E_{i}^{\prime}} S\right)+\eta(\operatorname{diam} S)^{k} .
$$

This decomposition will be instrumental in proving an analogue result, but for the packing dimension.

Lemma 20. If $f \in C^{\infty}\left(\mathbb{R}^{M}, \mathbb{R}^{N}\right)$, then the packing dimension of the critical set satisfies

$$
\operatorname{dim}_{P} f\left(C_{\kappa}\right) \leq \kappa .
$$

Proof. Let $k \geq 2$; since $f$ is of class $C^{\infty}, f$ is of class $C^{k}$. Let $\left\{A_{i}\right\}_{i \in \mathbb{N}}$ be the decomposition of $C_{\kappa}$ given by Lemma 19 with $\eta=1$. Let $K \subset \mathbb{R}^{M}$ be a compact set. Let $i \in \mathbb{N}$. Let $d_{i}$ be the distance in $\mathbb{R}^{M}$ such that for $x \in \mathbb{R}^{M}$ and $y \in \mathbb{R}^{M}$, $d_{i}(x, y)=d\left(\pi_{E_{i}^{\prime}} x, \pi_{E_{i}^{\prime}} y\right)+d\left(\pi_{E_{i}^{\prime \prime}} x, \pi_{E_{i}^{\prime \prime}} y\right)$. Let $\varepsilon>0$ and $\left\{B\left(f\left(x_{j}\right), r_{j}\right)\right\}_{j \in J}$ be a $\varepsilon$-packing of $f\left(A_{i} \cap K\right)$. Let $j \in J$ and $l \in J$. Taking $S:=\left\{x_{j}, x_{l}\right\}$ in (24) gives

$$
\begin{aligned}
r_{j}+r_{l} & \leq d_{i}\left(f\left(x_{j}\right), f\left(x_{l}\right)\right)=\operatorname{diam}\left(f\left(S \cap A_{i}\right)\right) \\
& \leq C d\left(\pi_{E_{i}^{\prime}} x_{j}, \pi_{E_{i}^{\prime}} x_{l}\right)+\left(d\left(\pi_{E_{i}^{\prime}} x_{j}, \pi_{E_{i}^{\prime}} x_{l}\right)+d\left(\pi_{E_{i}^{\prime \prime}} x_{j}, \pi_{E_{i}^{\prime \prime}} x_{l}\right)\right)^{k},
\end{aligned}
$$

where $C:=\|f\|_{C^{1}}+1$. This implies that

- either $C d\left(\pi_{E_{i}^{\prime}} x_{j}, \pi_{E_{i}^{\prime}} x_{l}\right) \geq \frac{1}{2}\left(r_{j}+r_{l}\right)$

- or $C d\left(\pi_{E_{i}^{\prime}} x_{j}, \pi_{E_{i}^{\prime}} x_{l}\right) \leq \frac{1}{2}\left(r_{j}+r_{l}\right)$, and then

$$
\left(d\left(\pi_{E_{i}^{\prime}} x_{j}, \pi_{E_{i}^{\prime}} x_{l}\right)+d\left(\pi_{E_{i}^{\prime \prime}} x_{j}, \pi_{E_{i}^{\prime \prime}} x_{l}\right)\right)^{k} \geq \frac{1}{2}\left(r_{j}+r_{l}\right) .
$$

Taking $\varepsilon$ so small that $\frac{\varepsilon}{C} \leq\left(1-2^{-1 / k}\right) \varepsilon^{1 / k}$, we get

$$
\frac{1}{2 C}\left(r_{j}+r_{l}\right) \leq\left(1-2^{-1 / k}\right)\left(\frac{1}{2}\left(r_{j}+r_{l}\right)\right)^{1 / k} ;
$$

thus

$$
d\left(\pi_{E_{i}^{\prime \prime}} x_{j}, \pi_{E_{i}^{\prime \prime}} x_{l}\right) \geq\left(\frac{1}{2}\right)^{1 / k}\left(\frac{1}{2}\left(r_{j}+r_{l}\right)\right)^{1 / k} .
$$


For $j \in J$, let $S_{j}=B_{E_{i}^{\prime}}\left(\pi_{E_{i}^{\prime}} x_{j}, \frac{1}{4 C} r_{j}\right) \times B_{E_{i}^{\prime \prime}}\left(\pi_{E_{i}^{\prime \prime}} x_{j}, \frac{1}{2}\left(\frac{1}{4} r_{j}\right)^{1 / k}\right) \subset E_{i}^{\prime} \times E_{i}^{\prime \prime}$. If $l \neq j$, we have $S_{j} \cap S_{l}=\emptyset$ since

- either $d\left(\pi_{E_{i}^{\prime}} x_{j}, \pi_{E_{i}^{\prime}} x_{l}\right) \geq \frac{1}{2 C}\left(r_{j}+r_{l}\right)>\frac{1}{4 C} r_{j}+\frac{1}{4 C} r_{l}$

- or $d\left(\pi_{E_{i}^{\prime \prime}} x_{j}, \pi_{E_{i}^{\prime \prime}} x_{l}\right) \geq\left(\frac{1}{4}\left(r_{j}+r_{l}\right)\right)^{1 / k}>\frac{1}{2}\left(\frac{1}{4} r_{j}\right)^{1 / k}+\frac{1}{2}\left(\frac{1}{4} r_{l}\right)^{1 / k}$.

There exists a constant $\delta_{i}$ such that

$$
\operatorname{diam} S_{j} \leq \delta_{i} \varepsilon^{1 / k}
$$

The rectangles $S_{j}$ are disjoints and have non-empty intersection with $K$, thus

$$
\sum_{j \in J} \operatorname{Vol}\left(S_{j}\right) \leq \operatorname{Vol}\left(K+B\left(0, \delta_{i} \varepsilon^{1 / k}\right)\right) .
$$

Let $p=\operatorname{dim} E_{i}^{\prime} \leq \kappa$. There exists a constant $\gamma_{i}$ such that the volume of each $S_{j}$ is

$$
\operatorname{Vol}\left(S_{j}\right)=\gamma_{i}\left(\frac{1}{4 C} r_{j}\right)^{p} \times\left[\frac{1}{2}\left(\frac{1}{4} r_{j}\right)^{1 / k}\right]^{M-p} .
$$

This implies, together with (25),

$$
\sum_{j \in J}\left(r_{j}\right)^{p+(M-p) / k} \leq c(i, k, K)<\infty,
$$

where $c(i, k, K)$ is a finite constant depending on $i, k$ and $K$.

Now, by definition of the $s$-dimensional packing measure (19) we obtain

$$
\mathcal{P}^{M / k+p(1-1 / k)}\left(f\left(A_{i} \cap K\right)\right) \leq c(i, k, K)<\infty .
$$

Therefore,

$$
\operatorname{dim}_{P} f\left(A_{i} \cap K\right) \leq \frac{M}{k}+p\left(1-\frac{1}{k}\right) \leq \frac{M}{k}+\kappa .
$$

Finally, taking a sequence of compacts $K_{n}$ such that $\mathbb{R}^{M}=\bigcup_{n \in \mathbb{N}} K_{n}$, we obtain

$$
\begin{aligned}
\operatorname{dim}_{P} f\left(C_{\kappa}\right) & =\operatorname{dim}_{P} f\left(\bigcup_{i, n \in \mathbb{N}} A_{i} \cap K_{n}\right) \\
& =\operatorname{dim}_{P} \bigcup f\left(A_{i} \cap K_{n}\right) \\
& =\sup _{i, n} \operatorname{dim}_{P} f\left(A_{i} \cap K_{n}\right) \quad \text { see [9] } \\
& \leq \frac{M}{k}+\kappa .
\end{aligned}
$$

Since $k$ is arbitrarily large we get

$$
\operatorname{dim}_{P} f\left(C_{\kappa}\right) \leq \kappa
$$

Without loss of generality, we prove Theorem 9 on $\mathbb{R}^{M}$ with $\mu$ equal to the Lebesgue measure $\lambda$. The general case can be deduced from it easily.

Proof of Theorem 9. Let $\kappa \in\{0, \ldots, \min \{M, N\}\}$.

1. If $A \subset C_{\kappa}$ and $\lambda(A)>0$, then $\operatorname{dim}_{H} f_{*}\left(\left.\lambda\right|_{A}\right) \geq \kappa$ :

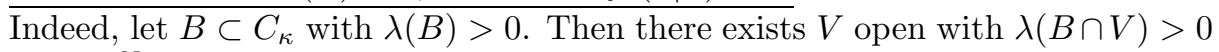
and $\pi: \mathbb{R}^{N} \rightarrow \mathbb{R}^{\kappa}$ a linear map such that $d_{x} \pi \circ f$ is of maximal rank $\kappa$ for every 
$x \in V$, and so $f_{\kappa}:=\pi \circ f$ satisfies $J f_{\kappa} \neq 0$ for every $x \in V$ (where $J$ is the Jacobian, i.e. $\left.J f_{\kappa}=\sqrt{\operatorname{det}\left(d_{x} f_{\kappa}\right)\left(d_{x} f_{\kappa}\right)^{t}}\right)$. Since $\pi$ is Lipschitz, it is known [9] that

$$
\operatorname{dim}_{H} \pi(f(B)) \leq \operatorname{dim}_{H} f(B) .
$$

Using the coarea formula (e.g. [8]; in fact we could have worked directly with $f$ using [10])

$$
\int_{B} J f_{\kappa} d \lambda=\int_{f_{\kappa}(B)} H^{M-\kappa}\left(B \cap f_{\kappa}^{-1}(\{y\})\right) d \lambda_{\kappa}(y),
$$

where $\lambda_{\kappa}$ is the Lebesgue measure on $\mathbb{R}^{\kappa}$. Since $\lambda(B \cap V)>0$ and $J f_{\kappa}(x) \neq 0$ for every $x \in B \cap V$, the left-hand side of (28) does not vanish and, therefore, neither does the right-hand side. Then $\lambda_{\kappa}\left(f_{\kappa}(B)\right)>0$ and so $\operatorname{dim}_{H} f_{\kappa}(B) \geq \kappa$, which gives, using (27),

$$
\kappa \leq \operatorname{dim}_{H} f(B) .
$$

Let $A \subset C_{\kappa}$ with $\lambda(A)>0$. We recall that

$$
\operatorname{dim}_{H} f_{*}\left(\left.\lambda\right|_{A}\right):=\inf \left\{\operatorname{dim}_{H} Y:\left(f_{*}\left(\left.\lambda\right|_{A}\right)\right)\left(Y^{c}\right)=0\right\} .
$$

Let $Y$ be such that $\left(f_{*}\left(\left.\lambda\right|_{A}\right)\right)\left(Y^{c}\right)=0$. Since $f\left(A \cap f^{-1}(Y)\right) \subset Y$ we have

$$
\operatorname{dim}_{H} Y \geq \operatorname{dim}_{H} f\left(A \cap f^{-1}(Y)\right) .
$$

Moreover, since $\lambda\left(f^{-1}(Y) \cap A\right)=\lambda(A)>0$ and $f^{-1}(Y) \cap A \subset C_{\kappa}$, we can choose $B=f^{-1}(Y) \cap A$ in the previous consideration and (29) gives

$$
\operatorname{dim}_{H} Y \geq \operatorname{dim}_{H} f\left(A \cap f^{-1}(Y)\right) \geq \kappa,
$$

and then

$$
\operatorname{dim}_{H} f_{*}\left(\left.\lambda\right|_{A}\right) \geq \kappa
$$

We define $\nu:=f_{*} \lambda$ and $\nu_{\kappa}:=f_{*}\left(\left.\lambda\right|_{C_{\kappa}}\right)$.

2. Let us prove that $d_{\nu}=\kappa \nu_{\kappa}$-almost everywhere:

- First, since $\nu_{\kappa}$ is supported by $f\left(C_{\kappa}\right)$, by Lemma 20 we have

$$
\operatorname{dim}_{P} \nu_{\kappa} \leq \operatorname{dim}_{P} f\left(C_{\kappa}\right) \leq \kappa .
$$

Since the packing dimension satisfies the relation (23), we get

$$
\bar{d}_{\nu_{\kappa}}(x) \leq \kappa \text { for } \nu_{\kappa} \text {-almost every } x \in \mathbb{R}^{N} \text {. }
$$

Since, for every $x \in \mathbb{R}^{N}$ and every $\varepsilon>0, \nu(B(x, \varepsilon)) \geq \nu_{\kappa}(B(x, \varepsilon))$, we have

$$
\bar{d}_{\nu}(x) \leq \bar{d}_{\nu_{\kappa}}(x)
$$

and then

$$
\bar{d}_{\nu}(x) \leq \kappa \text { for } \nu_{\kappa} \text {-almost every } x \in \mathbb{R}^{N} .
$$

- Let $K \subset \mathbb{R}^{N}$ be a compact subset, let $\rho<\kappa$ and let $Z:=\left\{\underline{d}_{\nu} \leq \rho\right\} \cap K$. If $\nu_{\kappa}(Z)>0$, then $\lambda\left(C_{\kappa} \cap f^{-1}(Z)\right)>0$; thus by (31) we obtain $\operatorname{dim}_{H} f_{*}\left(\left.\lambda\right|_{C_{\kappa} \cap f^{-1}(Z)}\right)$ $\geq \kappa$. By (30) and since $f_{*}\left(\left.\lambda\right|_{C_{\kappa} \cap f^{-1}(Z)}\right)\left(Z^{c}\right)=0$, we obtain $\operatorname{dim}_{H} Z \geq \kappa$.

On the other hand, by definition of $\underline{d}_{\nu}, \forall x \in Z, \exists J_{x} \subset(0,+\infty)$ with $0 \in \overline{J_{x}}$, such that $\forall r \in J_{x}, \nu(B(x, r)) \geq r^{\rho}$. Let $\delta>0$. We notice that $\{B(x, r), x \in Z, r \in$ $\left.J_{x} \cap[0, \delta]\right\}$ cover $Z$ so, by Besicovitch covering Theorem, there exists a subcovering $\left\{B\left(x_{i}, r_{i}\right)\right\}_{i \in I}$ with $I$ countable and $m_{0}$ a constant depending only on $N$ such that 
$Z \subset \bigcup_{i \in I} B\left(x_{i}, r_{i}\right)$ and the multiplicity of the subcovering is bounded by $m_{0}$. By the definition of (15)

$$
\mathcal{H}_{\delta}^{\rho}(Z) \leq \sum_{i \in I} r_{i}^{\rho} \leq \sum_{i \in I} \nu\left(B\left(x_{i}, r_{i}\right)\right) \leq m_{0} \nu(K+B(0, \delta)) .
$$

This implies $\operatorname{dim}_{H} Z \leq \rho$. But this is in contradiction with the fact that $\operatorname{dim}_{H} Z \geq$ $\kappa$. Then, for all $\rho<\kappa$ and for all compact $K, \nu_{\kappa}\left(\left\{\underline{d}_{\nu} \leq \rho\right\} \cap K\right)=0$. Thus

$$
\underline{d}_{\nu}(x) \geq \kappa \text { for } \nu_{\kappa} \text {-almost every } x \in \mathbb{R}^{N} \text {. }
$$

3. Conclusion:

Using (32) together with (33) implies that $d_{\nu}=\kappa \nu_{\kappa}$-almost everywhere. The theorem follows from $\mathbb{R}^{M}=C_{0} \cup C_{1} \cup \ldots \cup C_{\min \{M, N\}}$.

\section{REFERENCES}

1. Viviane Baladi, Positive transfer operators and decay of correlations, Advanced Series in Nonlinear Dynamics, vol. 16, World Scientific Publishing Co. Inc., River Edge, NJ, 2000. MR:1793194 (2001k:37035)

2. L. Barreira and B. Saussol, Hausdorff dimension of measures via Poincaré recurrence, Comm. Math. Phys. 219 (2001), no. 2, 443-463. MR.1833809 (2002c:37035)

3. Sean M. Bates and Carlos G. Moreira, De nouvelles perspectives sur le théorème de MorseSard, C. R. Acad. Sci. Paris Sér. I Math. 332 (2001), no. 1, 13-17. MR1805620(2002b:58010)

4. Michael D. Boshernitzan, Quantitative recurrence results, Invent. Math. 113 (1993), no. 3, 617-631. MR 1231839 (94k:28028)

5. N. Chernov, Statistical properties of piecewise smooth hyperbolic systems in high dimensions, Discrete Contin. Dynam. Systems 5 (1999), no. 2, 425-448. MR1665752 (99k:58134)

6. M. Maurice Dodson and Simon Kristensen, Hausdorff dimension and Diophantine approximation, Fractal geometry and applications: A jubilee of Benoît Mandelbrot. Part 1, Proc. Sympos. Pure Math., vol. 72, Amer. Math. Soc., Providence, RI, 2004, pp. 305-347. MR 2112110 (2005m:11139)

7. Dmitry Dolgopyat, On mixing properties of compact group extensions of hyperbolic systems, Israel J. Math. 130 (2002), 157-205. MR1919377 (2003m:37037)

8. Lawrence C. Evans and Ronald F. Gariepy, Measure theory and fine properties of functions, Studies in Advanced Mathematics, CRC Press, Boca Raton, FL, 1992. MR 1158660 (93f:28001)

9. Kenneth Falconer, Techniques in fractal geometry, John Wiley \& Sons Ltd., Chichester, 1997. MR 1449135 (99f:28013)

10. Makoto Ohtsuka, Area formula, Bull. Inst. Math. Acad. Sinica 6 (1978), no. 2, part 2, 599-636. MR528671 (80a:28003)

11. William Ott and James A. Yorke, Learning about reality from observation, SIAM J. Appl. Dyn. Syst. 2 (2003), no. 3, 297-322 (electronic). MR2031277 (2004m:37041)

12. Benoît Saussol, Recurrence rate in rapidly mixing dynamical systems, Discrete Contin. Dyn. Syst. 15 (2006), no. 1, 259-267. MR2191396 (2006k:37005)

13. Lai-Sang Young, Statistical properties of dynamical systems with some hyperbolicity, Ann. of Math. (2) 147 (1998), no. 3, 585-650. MR.1637655 (99h:58140)

Université Européenne de Bretagne, Université de Brest, Laboratoire de Mathématiques UMR CNRS 6205, 6 AVenue Victor le Gorgeu, CS93837, F-29238 Brest Cedex 3 FRANCE

E-mail address: jerome.rousseau@univ-brest.fr

Université Européenne de Bretagne, Université de Brest, Laboratoire de Mathématiques UMR CNRS 6205, 6 avenue Victor le Gorgeu, CS93837, F-29238 Brest Cedex 3 FRANCE

E-mail address: benoit.saussol@univ-brest.fr 